\title{
SUMS OF FUNCTIONS OF BOUNDED INDEX
}

\author{
WALTER PUGH ${ }^{1}$
}

Introduction. The notion of entire functions of bounded index has been studied by several authors in a number of recent papers [1], [2], [3], [4].

Little is known about the properties of such functions, and, in particular, the following "natural" question (which is answered in this note) does not appear to have been studied.

Is the sum of two functions of bounded index also of bounded index?

Let $g(z)$ be an entire function. We write as usual

$$
M(\Gamma, g)=\max _{|z|=\Gamma}|g(z)|,
$$

and given the integer $\nu \geqq 0$ we associate with $\nu$ and $g$ the nonnegative function

$$
\Omega(z)=\Omega_{\nu}(z, g)=\max _{0 \leqq \delta \leqq \nu}\left\{\frac{\left|g^{(z)}(z)\right|}{s !}\right\} .
$$

If $g(z)$ is of bounded index $\nu$, we have, by definition

$$
\left|\frac{g^{(n)}(z)}{n !}\right| \leqq \Omega(z)
$$

for all $z$ and all integers $n \geqq 0$.

It is easily seen that there exist functions of index $\nu<+\infty$ such that (2) may be replaced by

$$
\left|\frac{g^{(n)}(z)}{n !}\right| \leqq \alpha \Omega(z) \quad(n=\nu+1, \nu+2, \nu+3, \cdots ; 0<\alpha<1),
$$

where $\alpha$ is a constant.

Using the above definitions and notation the author proves the following

ThEOREM. Let $g(z)$ be a function of index $\nu<+\infty$ satisfying the condition (3).

Let $f(z)$ be any entire function such that

Received by the editors October 21, 1968.

1 The author gratefully acknowledges support by the National Science Foundation under grant GP-7507.

This paper is being published posthumously. Professor Pugh died April 17, 1969. The galley proof was proofread and corrected by Professor S. M. Shah of the University of Kentucky. 


$$
f(0) \neq 0,
$$

and such that

$$
M(2|z|, f) \leqq \Omega(z)
$$

for all $z$.

Then, if the constant $c(\neq 0)$ is chosen small enough, the function

$$
h(z)=g(z)+c f(z)
$$

is of index not greater than $\nu$.

If for all positive values of $q$

$$
\lim _{|z| \rightarrow \infty} \frac{\Omega(z)}{|z|^{q}}=+\infty,
$$

and if $\Omega(z)$ never vanishes, it is easy to find an entire function $f(z)$, of unbounded index satisfying the conditions (4) and (5).

Let $f(z)$ be such a function. Then, in view of the theorem, a suitable choice of $c$ yields

$$
F(z)=c f(z)=h(z)-g(z) .
$$

Here $F(z)$ is of unbounded index whereas $h(z)$ and $-g(z)$ are of index at most $\nu(<+\infty)$.

We thus obtain the

COROLLARY. The sum of two functions of bounded index need not be of bounded index.

1. Proof of Theorem 1. If $f(z)$ is entire, Cauchy's estimate yields

$$
\left|\frac{f^{(n)}(z)}{n !}\right| \Gamma^{n} \leqq M(2 \Gamma, f) \quad\left(|z|=\Gamma, f^{(0)}(z) \equiv f(z)\right)
$$

for all $z$ and all integers $n \geqq 0$.

From (1.1) we deduce

$$
\left|\frac{f^{(n)}(z)}{n !}\right| \leqq M(2, f) \quad(|z| \leqq 1) .
$$

By (4) $B=M(2, f) /|f(0)| \geqq 1$, and, hence (1.1) and (1.2) yield

$$
\left|\frac{f^{(n)}(z)}{n !}\right| \leqq B M(2 \Gamma, f) \quad(|z|=\Gamma)
$$

for all $z$ and all integers $n \geqq 0$. 
Let $g(z)$ and $f(z)$ satisfy the conditions of the theorem. We differentiate (6) $n \geqq \nu+1$ times and then use (3), (1.3), and (5); this yields

$$
\left|\frac{h^{(n)}(z)}{n !}\right| \leqq \alpha \Omega(z)+|c| B M(2 \Gamma, f) \leqq \Omega(z)(\alpha+|c| B)
$$

$$
(n \geqq \nu+1) .
$$

If $s \leqq \nu,(1.3)$ is still valid with $n$ replaced by $s$, but (3) no longer holds, and the differentiation of (6) only leads to

$$
\left|\frac{h^{(s)}(z)}{s !}\right| \geqq\left|\frac{g^{(s)}(z)}{s !}\right|-|c| B M(2 \Gamma, f) \quad(0 \leqq s \leqq \nu) .
$$

In view of the definition (1) we see that (1.5) and (5) imply

$$
\max _{0 \leqq s \leqq \nu}\left\{\left|\frac{h^{(s)}(z)}{s !}\right|\right\} \geqq \Omega(z)-|c| B \Omega(z) .
$$

If $|c| B<1$, we deduce from (1.4) and (1.6)

$$
\begin{aligned}
\left|\frac{h^{(n)}(z)}{n !}\right| \leqq \frac{\alpha+|c| B}{1-|c| B} \max _{0 \leqq s \leqq \nu}\left\{\left|\frac{h^{(s)}(z)}{s !}\right|\right\} \\
(n=\nu+1, \nu+2, \nu+3, \cdots) .
\end{aligned}
$$

As $c \rightarrow 0$

$$
\lambda=\frac{\alpha+|c| B}{1-|c| B} \rightarrow \alpha<1
$$

Hence, if $c$ is small enough, $\lambda \leqq 1$ and (1.7) shows that $h(z)$ is of index at most equal to $\nu<+\infty$. This completes the proof of Theorem 1 .

2. Proof of the Corollary. The function

$$
g(z)=\cos z+\cosh z
$$

satisfies the differential equation $g^{(4)}(z)=g(z)$, and, hence, if we set

$$
\max _{0 \leqq s \leqq 3}\left\{\left|\frac{g^{(s)}(z)}{s !}\right|\right\}=\Omega(z)
$$

we obtain

$$
\left|\frac{g^{(n)}(z)}{n !}\right|=\frac{k !}{n !}\left|\frac{g^{(k)}(z)}{k !}\right| \leqq \frac{\Omega(z)}{4} \quad(n \geqq 4),
$$

where $k \equiv n(\bmod 4)$ and $0 \leqq k \leqq 3$. 
Lemma. Let $g(z)$ be defined by (2.1) and $\Omega(z)$ by (2.2). Then, for all $z$,

$$
\Omega(z) \geqq A e^{|z| / 2} \quad(0<A=\text { const }) .
$$

Proof. It is obvious that $\Omega(z)$ is a continuous function of $z$, and that, in view of the differential equation, $\Omega(z)$ never vanishes. Hence, by choosing $A$ sufficiently small, we can always assume that (2.4) holds for $|z| \leqq 2$.

Now

$|\cosh z|^{2}=\frac{1}{2}(\cosh 2 x+\cos 2 y) \geqq \frac{1}{4}\left(e^{2 x}+e^{-2 x}-2\right) \quad z=(x+i y)$,

and hence

$$
|\cosh z| \geqq A e^{|x|} \quad(0<A=\text { const } ;|x| \geqq 1) .
$$

(Throughout this proof we denote positive constants by $A$ and we do not assume that $A$ has the same value each time it occurs.)

From (2.5) we deduce

$$
|\cos z|=|\cosh i z| \geqq A e^{|y|} \quad(|y| \geqq 1) .
$$

$$
\text { If }|z| \geqq 2
$$

$$
\max \{|x|,|y|\} \geqq|z| / 2 \geqq 1
$$

and, by (2.5) and (2.6),

$$
|\cosh z|+|\cos z| \geqq A e^{|z| / 2} \quad(|z| \geqq 2) .
$$

From (2.1) we deduce

$$
2 \cosh z=g+g^{\prime \prime}, \quad 2 \cos z=g-g^{\prime \prime},
$$

and, in view of (2.7)

$$
\begin{aligned}
A e^{|z| / 2} \leqq|\cos z|+|\cosh z| \leqq 4 \max \left\{|g|,\left|\frac{g^{\prime \prime}}{2}\right|\right\} \leqq & 4 \Omega(z) \\
& (|z| \geqq 2) .
\end{aligned}
$$

This proves the lemma because we have shown already that (2.4) holds for $|z| \leqq 2$.

The function $\phi(z)=\coprod_{j=1}^{\infty}\left(1+z / e^{j}\right)^{j}$ is of unbounded index (because it has zeros of arbitrarily high multiplicity) and is clearly of order zero. Hence, in view of (2.4)

$$
M(2 \Gamma, \phi) / \Omega(z) \leqq L, \quad(|z|=\Gamma)
$$

where $L$ is a finite bound. We set

$$
f(z)=\frac{1}{L} \phi(z)
$$


and notice that, in view of (2.3) and (2.8) the theorem is applicable to the functions $g(z)$ and $f(z)$ defined by (2.1) and (2.9). Hence, for small $c$,

$$
F(z)=c f(z)=\frac{c}{L} \phi(z)
$$

is a function of unbounded index which may be represented as the sum of two functions of bounded index.

The author gratefully acknowledges the help in the writing of this paper given by Professor Albert Edrei.

\section{REFERENCES}

1. B. Lepson, Differential equations of infinite order, hyperdirichlet series, and entire functions of bounded index, Proc. Sympos. Pure Math., vol. 11, Amer. Math. Soc., Providence, R.I., 1968, pp. 298-307.

2. W. J. Pugh and S. M. Shah, On the growth of entire functions of bounded index, (to appear).

3. S. M. Shah, Entire functions of bounded index, Proc. Amer. Math. Soc. 19 (1968), 1017-1022.

4. - Entire functions satisfying a linear differential equation, J. Math. Mech. 18 (1968), 131-136.

SYRACUSE UNIVERSITY 